J3eA, Journal sur l'enseignement des sciences et technologies de l'information et des systèmes, Volume 4, Hors-Série 3, 1 (2005)

DOI : http://dx.doi.org/10.1051/bib-j3ea:2005801

(C) EDP Sciences, 2005

\title{
Coded light projection techniques for 3D reconstruction
}

\author{
J. Pagès and J. Salvi \\ Institute of Informatics and Applications \\ University of Girona \\ Av. Lluís Santaló s/n \\ SP-17071 Girona, Spain
}




\title{
CODED LIGHT PROJECTION TECHNIQUES FOR 3D RECONSTRUCTION
}

\author{
Jordi Pagès and Joaquim Salvi \\ Institute of Informatics and Applications \\ University of Girona \\ Av. Lluís Santaló s/n, 17071 Girona (Spain)
}

\begin{abstract}
Coded structured light is a computer vision technique which aims to reconstruct objects. This kind of technique belongs to the group of active triangulation methods. The setup usually consists of a LCD projector and a camera. The LCD device projects a pattern or a set of patterns so that finding correspondences between the projected and the viewed images becomes easier. The coding strategy used to generate the patterns determines the performance of the system in terms of resolution (number of encoded points) and accuracy. This paper presents a classification of the existing techniques as well as a new coding strategy which aims to optimise classic one-shot patterns based on De Bruijn codification.
\end{abstract}

\section{INTRODUCTION}

Object reconstruction is one of the most important topics in computer vision due to its wide field of application. Some examples of applications are range acquisition, industrial inspection, reverse engineering, object recognition and 3D map building. Amongst all the ranging techniques [13], stereovision is based on viewing the scene from two or more points of view and then finding correspondences between the different images in order to triangulate the $3 \mathrm{D}$ position. Triangulation is possible if cameras have been calibrated beforehand $[6,24]$. However, difficulties in finding the correspondences arise, even when taking into account epipolar constraints. Coded structured light consists of replacing one of the two cameras by a device that projects a light pattern onto the measuring surface. Nowadays the most commonly used devices are LCD video projectors. Such devices project an image with a certain structure so that a set of pixels are easily distinguishable by means of a local coding strategy. Therefore, when locating such coded points in the image grabbed by the remaining camera, the correspondence problem is solved with no requirement for geometrical constraints. The projecting images are called patterns, as they are globally structured.

Work funded by Spanish project CICYT TAP99-0443-C05-01
The first part of the paper (see section 2) exposes the main groups of coded light techniques and briefly summarises the most important coding strategies. Few experimental results are given in order to compare several techniques. Furthermore, some guidelines are presented in order to chose the more adequate technique depending on the goal and constraints of the reconstruction task.

The second part of the paper (see section 3), focuses on a certain type of coded light techniques called one-shot patterns, since a unique pattern is projected. A new coding strategy is presented. The advantages of such strategy over the existing ones are discussed and some experimental results are shown.

\section{CLASSIFICATION OF CODIFICATION STRATEGIES}

A coded structured light system is based on the projection of a single pattern or a set of patterns onto the object which is then viewed by a single camera or a set of cameras. The patterns are specially designed so that codewords are assigned to a set of pixels. Every coded pixel has its own codeword, so there is a direct mapping from the codewords to the corresponding coordinates of the pixel in the pattern. The codewords are mapped in the pattern by using grey levels, colour or geometrical representations. The larger the number of points that must be coded, the larger the codewords are and, therefore, the mapping of such codewords to a pattern is more difficult.

In Table 1 a large number of coded light techniques are classified according to their codification strategy. The features indicating if a pattern is suitable to measure moving objects or not, the considered colour depth and whether repeated codewords appear (periodical coding) or not (absolute coding) are all indicated in the seven columns at the right side of the table.

The first division among techniques differentiates between patterns based on temporal (time-multiplexing), spatial and direct codification. Time-multiplexing strategy is based on assigning a sequence of values to every coded point by projecting a set of patterns along time. Spatial cod- 
ification refers to those patterns whose points are encoded with the information included in a neighbourhood around them. Direct codification means that each point of the pattern is identified just by its own value (grey level or colour). In the following subsections every subgroup of techniques

Table 1. The proposed classification

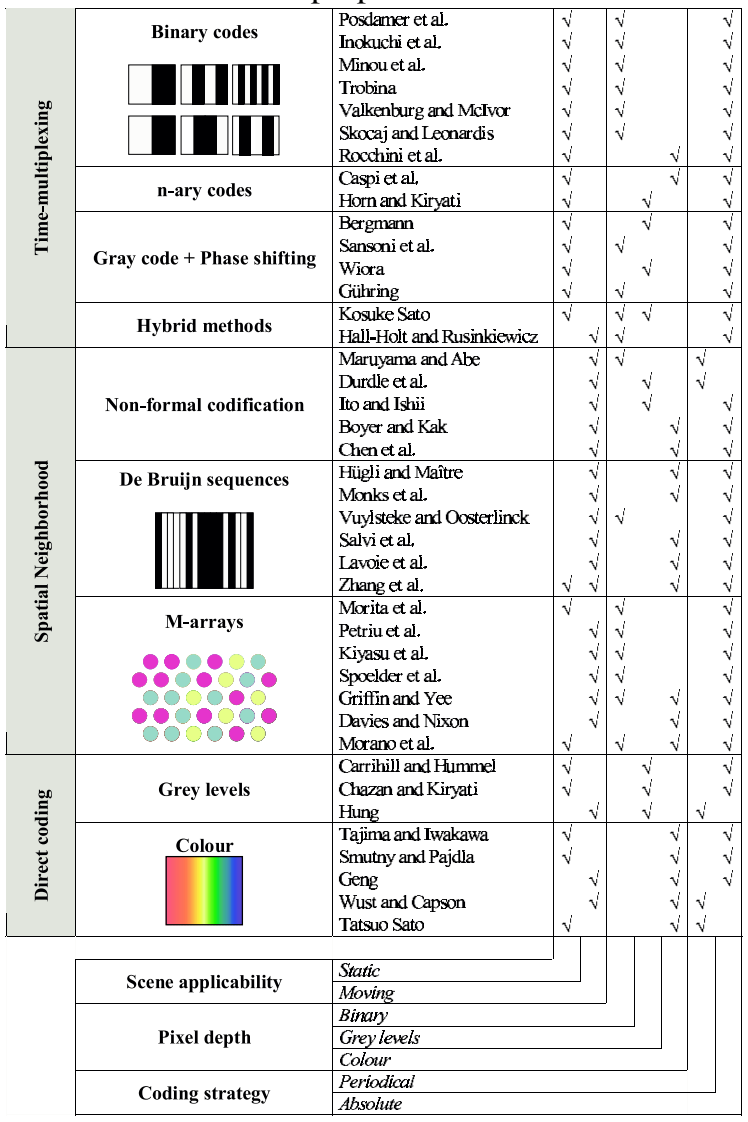

are briefly explained. A more comprehensive survey can be found in [26].

\subsection{Time-multiplexing}

The former coded light techniques were based on temporal coding. In this case, a set of patterns are successively projected onto the object. The codeword for a given point is usually formed by the sequence of the illuminance values received by such point. The main constraint of temporal systems is their inapplicability to dynamic scenes. This kind of patterns can reach a high accuracy in the measurements. This fact relies on two points: first, a small set of colours or grey levels are used, so they can easily be binarised or segmented; second, a coarse-to-fine paradigm is followed, since the position of a pixel is being encoded more precisely by the time the patterns are projected. In addition, large resolution can be achieved.

\subsubsection{Patterns based on binary codes}

Black and white are used to encode every pattern point. Stripe codification is usually used in these approaches. A stripe is a band of adjacent pixels sharing the same colour. Posdamer et al. [23] were the first who proposed the projection of a sequence of $m$ patterns to encode $2^{m}$ stripes using a plain binary code. Thus, the codeword associated to each pixel is the sequence of $0 \mathrm{~s}$ and $1 \mathrm{~s}$ obtained from the $m$ patterns. Valkenburg and McIvor [29] decreased the measuring error using Gray code and estimating the position of the stripes with subpixel accuracy. An example of the initial binary patterns of a sequence encoded with Gray code is shown in Fig. 1a. Minou et al. [18] proposed the use of Hamming code in order to detect errors and even correcting them increasing the length of the codewords, i.e. the number of projected patterns.

\subsubsection{Patterns based on n-ary codes}

This group of techniques use an extension of the binary Gray code so that an alphabet $n$ symbols is used. By increasing the basis of the code, the number of required patterns decreases. Caspi et al. [3] mapped every symbol of the alphabet to a certain colour, while Horn and Kiryati [10] mapped every symbol to a certain grey level. In general, $n$-ary techniques are useful to obtain similar or even better performance that with binary codes, but reducing the number of patterns.

\subsubsection{Gray code and Phase shifting}

This methods are based on the classical line stripe scanning over time. Laser scanners are based on scanning the object by projecting a light stripe and taking an image for every position of the laser onto the object surface. Knowing each laser projection angle, triangulation can be fulfilled for every imaged stripe position. In order to minimise the scanning time, some authors use a slide projector to project a multi-stripe pattern onto the scene. In order to distinguish among all the projected stripes, several patterns of Gray code are also projected in order to label every region scanned by each stripe. An important work in this field was presented by Gühgring [8] obtaining great results.

These techniques can obtain high accuracy in spite of a large number of patterns.

\subsubsection{Hybbrid methods}

There are some techniques mainly based on the projection of multiple encoded patterns along time. However, if they also take into account a small spatial neighbourhood of pattern points in the codification strategy they can be considered as hybrid methods. 
Hall-Holt and Rusinkiewicz [9] divided four patterns in a total of 111 vertical stripes that are painted in white or black. Codification is located at the edges between each pair of stripes. The codeword of each edge is formed by 8 bits. Every pattern gives 2 of these bits, representing the value of the bounding stripes. Therefore, the technique used timemultiplexing (since 4 coded patterns are projected) and also spatial coding (a neighbourhood of 2 stripes is considered in every pattern). In fact, this technique is a variation of classic binary patterns which reduces the number of projections by taking into account a small neighbourhood.

\subsection{Spatial neighbourhood}

This subgroup of techniques tend to concentrate all the coding scheme in a unique pattern. The codeword that labels each pixel is obtained from a neighbourhood of pixels around it. In most cases they are suitable for measuring moving surfaces. However, the decoding stage is more difficult because the whole neighbourhood can not always be recovered due to occlusions and shadows. The following subsections summarise the most common codification theories used for spatial coding.

\subsubsection{Non-formal codification}

The first authors exploiting spatial neighbourhood did not use any formal codification theory in order to define the codewords represented in every neighbourhood. Such patterns were usually generated by brute-force algorithms accomplishing some constraints. Different techniques belonging to this group can be found in the bibliography. They basically differ in the visual features used to identify every neighbourhood of the patterns.

Some works belonging to this group are the multi-slit coloured pattern by Boyer and Kak [1], the grid pattern painted in grey levels by Ito [12] and the multi-stripe coloured pattern by Chen et al. [4]. An original pattern was presented by Maruyama and Abe [17]. The pattern was composed of vertical slits containing random cuts. The position of all the cuts of a given slit differentiates that slit from all the rest.

\subsubsection{Patterns encoded with De Bruijn sequences}

The techniques belonging to the previous subsection had lack of robustness since repeated neighbourhoods could appear, i.e. uniqueness of codewords is not ensured. This problem was solved for some authors who realised that $D e$ Bruijn sequences are very useful to encode patterns based on spatial neighbourhood. A De Bruijn or pseudorandom sequence of order $m$ over an alphabet of $n$ symbols is a circular string of length $n^{m}$ that contains each substring of length $m$ exactly once [16]. This sort of sequences can be obtained searching Eulerian cycles or Hamiltonian cycles over different kinds of De Bruijn graphs.

Pseudorandom sequences have been used for encoding multi-slit (narrow stripes separated by black gaps) [11, 19], multi-stripe (adjacent stripes) [31] and grid patterns [15,25] among others [30].

\subsubsection{Patterns encoded with M-arrays}

The extension of the pseudorandom sequences to the bidimensional case has also been used to encode patterns. Let $M$ be a matrix of dimensions $r \times v$ where each element is taken from an alphabet of $k$ symbols. If $M$ has the window property, i.e. each different window of dimensions $n \times m$ appears exactly once, then $M$ is a perfect map or a pseudorandom array. These kind of arrays have been widely used in pattern codification because the window property allows every different sub-matrix to be associated to an absolute position in the array.

Pseudorandom arrays have been largely used in order to encode patterns composed of binary and coloured dots $[5,7$, 20,21], grid patterns [14,22] and checkerboard patterns [27]. In Fig. 1d an example of dot pattern is shown.

\subsection{Direct codification}

There are techniques based on assigning to each coded pattern point a certain colour or grey level which identifies such point. These techniques usually suffer from two problems: either they have low resolution or they are very sensitive to noise or they have periodicity. If a large range of colours or grey levels are used in the same pattern (in order to encode a large number of points), the "distance" between codewords reduces and problems appear due to the non-linear response of the projector-camera system and the object albedo. Another possibility is that, in order to preserve a good resolution, the pattern is periodic so repeated codewords can produce false correspondences and erroneous reconstructions.

A classic technique in this group is the intensity ratio sensor by Carrihill and Hummel [2] where the pattern is a column gradient from black to white. Similarly, Tajima and Iwakawa [28] defined the rainbow pattern where a large set of stripes are coloured with a sampling sequence of the colour spectrum from red to blue. Sato presented a variation of the rainbow technique where the a periodic pattern is projected 3 times by shifting it $1 / 3$ of its period so that by using an adequate linear combination of the 3 images the albedo of the object can be canceled (assuming a constant reflection model). The patterns proposed by Sato are shown in Fig. 1e. 
a)

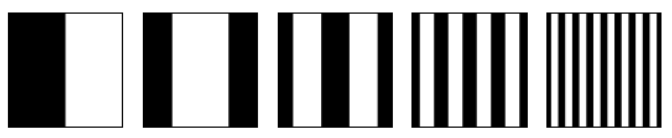

b)
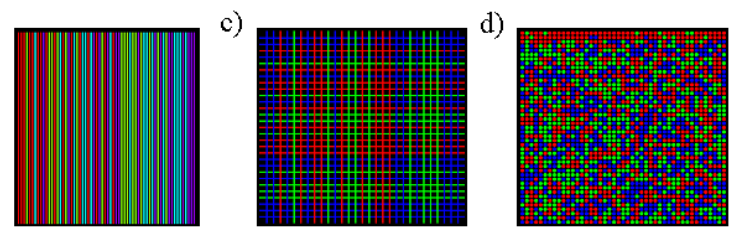

e)

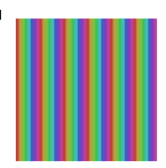

a)

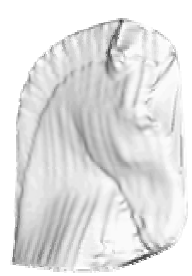

c)

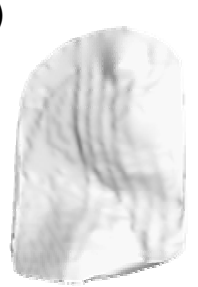

b)

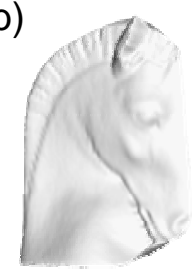

d)

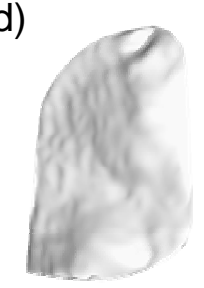

Fig. 1. Examples of patterns; a) Time-multiplexing based on a sequence of Gray coded patterns; b) Pattern of vertical colored slits encoded with a De Bruijn sequence; c) Grid pattern with De Bruijn sequence codification; d) Pattern of colored dots encoded with an M-array; e) Direct codification using three shifted periodic rainbow patterns.

\subsection{Some experimental results}

In Fig. 2 the results of reconstructing a white horse statue are presented. In Fig. $2 \mathrm{a}$ the reconstruction by using $7 \mathrm{bi}-$ nary Gray patterns is presented. As can be seen, the reconstruction has a good resolution but shows same periodic ridges which break the object smoothness. This is mainly due to the sub-pixel detection of edges between binary stripes. The exact location of an edge is affected by the intensity contrast between a black and a white stripe. This problem is solved by the technique of Gühring (see Fig. 2b), since the sub-pixel detection of a slit (intensity peak) is more accurate. Furthermore, since the slits are scanned over the object the resolution is higher in spite of projecting 14 patterns. The result of projecting a De Bruijn encoded pattern composed of 64 slits based on 4 colours is shown in Fig. 2c. We can see the lower resolution (since a unique pattern is projected) and some vertical ridges due to the sub-pixel location of the slits has not been optimised. Finally, in Fig. 2 we have the results when projecting an marray of $45 \times 45$ dots coloured with 3 colours. The resolution is, as expected, even worse.

\subsection{Conclusions of the state of the art}

Techniques based on time-multiplexing have generally demonstrated high accuracy, large resolution and an easy decoding stage as only a reduced set of colours is used (often black and white). However, its main constraint is their inapplicability to moving objects since multiple patterns must be projected. A way to solve this problem is to identify each pattern point by a certain spatial neighbourhood or increasing the number of colours used. The larger the number of colours, the smaller the required neighbourhood, in spite of a harder decoding complexity. When using spatial neighbourhood a unique pattern can be defined so that moving surfaces can be measured. However, discontinuities on the object can produce false identification of certain pattern regions. Moreover, since the codification is condensed in a unique pattern, the maximum resolution is lower. Another way to reduce the number of patterns is using the maximum grey level or colour spectrum, so that each column or row of the pattern has its own value. This solution, however, is only recommended when dealing with neutral colour scenes. It is necessary to find a trade off between the number of patterns to project and the number of colours or grey levels used to encode, which is also related to the acquisition time and the accuracy that can be obtained.

In summary, if the objective is to obtain high accuracy and dealing with static scenes, time-multiplexing is the most suitable approach. Moreover, the most complete techniques are the ones proposed by Caspi et al. [3] and Guhring [8]. When dealing with dynamic surfaces, the patterns based on M-arrays or pseudorandom sequences are recommended. The difficulty relies on designing a decoding algorithm robust enough to take into account discontinuities in the perceived pattern. The most robust solution to this problem has been proposed by Zhang et al. [31]. 


\section{A NEW ONE-SHOT PATTERN BASED ON DE BRUIJN CODING}

In this section we present a one-shot technique based on projecting a unique multi-stripe pattern encoded with De Bruijn sequences. A lot of similar patterns have been proposed in the bibliography. However, the aim of our work is to improve the coding strategy in order to maximise the resolution of the pattern (number of stripes) by using few colours.

The best stripe pattern that can be found corresponds to Zhang et al. [31] which is based on 125 adjacent stripes coloured using 8 different levels of Hue. Although the experimental results provided by this technique are very good, it is mainly due to a robust and complex decoding stage. However, we could expect to design a simpler technique by using a small number of colours. In the rest of the paper we present a new simple way to encode stripe patterns in order to reduce the number of colours by maintaining the same resolution.

\subsection{Classic stripe patterns}

The most typical way to encode a stripe pattern is to use a De Bruijn sequence based on $n$ symbols and a window property (order of the sequence) of $m$. Each symbol of the alphabet is mapped to a certain colour (Hue value). Thanks to the window property, each stripe is identified by any of the windows of length $m$ containing this stripe. An example of binary $(n=2)$ De Bruijn sequence of order $m=4$ is

$$
1000010111101001
$$

note that every substring of 4 elements appears only once in the sequence. With such a sequence a pattern consisting of $n^{m}=16$ binary stripes can be encoded assigning, for instance, black to 0 and white to 1 . However, note that in a De Bruijn sequence consecutive elements can be equal, and therefore, the corresponding pattern will have consecutive stripes sharing the same colour. This produces that these stripes appear as a single stripe. That is why when using a stripe pattern, the De Bruijn sequence must be constrained to not allow consecutive elements to have the same value. Hügli and Maître [11] studied such sequences and found that their resolution decreases to $n(n-1)^{m-1}$. It implies that if we want to increase the resolution of the stripe pattern we must increase the number of colours or the window size. Increasing the window size is not suitable since then it is more possible to violate the local smoothness assumption of the object. On the other hand, increasing the number of colours increases the sensitivity against noise, reflectance properties of the object and the non-linearities of the projector and the camera.

The technique by Zhang et al. [31] is based on 8 colours and a window property of 3 stripes. Note that projecting 8

colours can be very problematic depending on the application. We now present an alternative way to encode stripe patterns by using fewer colours and maintaining the resolution and the window size.

\subsection{New coding strategy}

The aim of our coding strategy is producing stripe patterns with a window property of length 3 , by using $n$ different Hue values.

The stripe pattern is defined so that the luminance profile of every row is a periodic square function based on two levels of luminance higher than 0 . This type of profile produces alternating maxima and minima luminance peaks when viewing the projected pattern from a camera. Then, the luminance value of the stripes is set alternatively from low to high luminance. Then, the pattern is divided into $n$ periods. All the full-illuminated stripes of a certain period are painted with the same Hue value. The Hue of the half-illuminated stripes of a period is chosen by using a De Bruijn sequence of order $m-1$ based on $n$ symbols. The same sequence is used for all the periods. Note that the resulting resolution is $2 n^{m}$ stripes in front of $n(n-1)^{m-1}$ (where $m=3$ ) which would be the one achieved using a constrained De Bruijn sequence for the whole pattern. It is possible because two consecutive stripes can share the same Hue value, but not the same luminance level.

In Fig. 3 an example of pattern encoded with this simple strategy is presented. In the example, the pattern has 128 stripes encoded with 4 Hue values. Note the periodic luminance profile. In the following subsections, some results produced by this pattern are presented.

a)

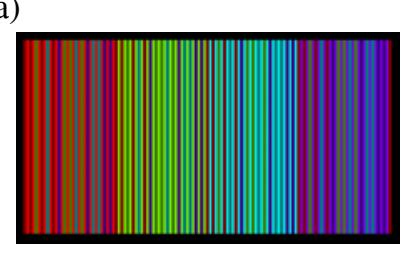

Fig. 3. The proposed pattern. a) RGB pattern. b) Luminance channel of the pattern

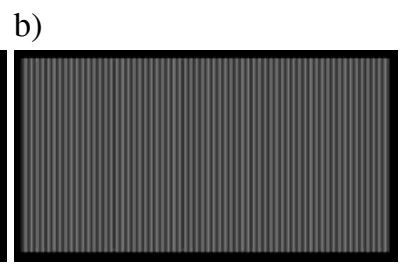

\subsection{Experimental results of the new pattern}

Our experimental setup consisted of an XGA LCD projector Mitsubishi XL1U working at $1024 \times 768$ pixels, a camera Sony $3 \mathrm{CCD}$, a Meteor II frame grabber digitising images of $768 \times 576$ pixels with 24-bits colour depth, and a standard PC.

The acquisition process consists of positioning the camera beside the projector, and the object to be reconstructed 
at a certain distance in front of the devices so that the projected pattern appears focused. The camera and the projector must be geometrically calibrated in order to triangulate correspondences. Then, by taking a single image from the camera, the decoding process must be fulfilled.

The decoding process starts by searching stripes in a set of rows of the image. Since a periodic luminance profile is projected, segmenting the stripes can be done by deriving the luminance profile of every row of the image. The derivatives are estimated by applying a linear filter of order $n$ like the one shown in equation 2. $f(i)$ is the grey level of the $i$ th pixel of the current row of the image.

$$
g(i)=\sum_{c=1}^{n / 2}(f(i+c)-f(i-c))
$$

In our case, a filter of order 8 is sufficient. The effects of applying this filter to the luminance channel of the images is shown in figure 4 . If the 2 nd derivative is binarised, the regions corresponding to full-illuminated stripes and halfilluminated stripes are located. Then, in every stripe region, the sub-pixel position of the maximum or minimum peak can be accurately calculated with the Blais and Rioux peak detector as described in [8]. Once all the stripes of

a)
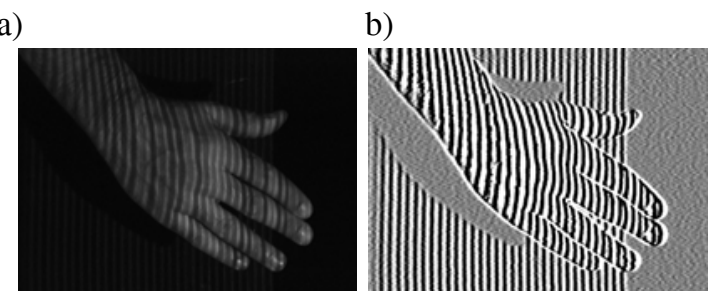

Fig. 4. 2nd Derivative of the luminance channel. a) Luminance image of a human hand under the pattern illumination. b) Result of applying the 2 nd derivative.

a given image row have been located, their colour can be easily identified since only 4 levels of Hue are projected. Afterwards, in order to match a given perceived stripe with the projected on it is only necessary to look at its own colour and the colours of the two adjacent stripes (window property equal to 3 ).

If this process is repeated for all the rows of the image, a large number of correspondences can be found, leading to a dense $3 D$ reconstruction of the object. Several examples are now presented.

A horse statue with smooth depth variation has been reconstructed as shown in figure 5a. The proposed technique preserves the smoothness of the object and, at the same time, it detects some small details like the horse eye. The sun statue has been used for testing the resolution of the new technique. As shown in figure 5b, the mouth, eyes and nose of the sun are easily appreciable, and even some details of the flames. The last test consisted of reconstructing a human hand, which presented some problems. The hand texture introduces a strong gain in the red channel of the images. However, thanks to the reduced number of colours projected they could be correctly identified and the surface was successfully reconstructed as shown in figure $5 \mathrm{c}$.

a)
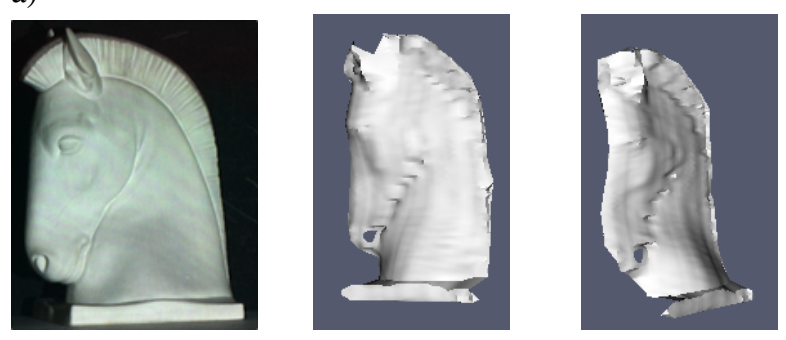

b)
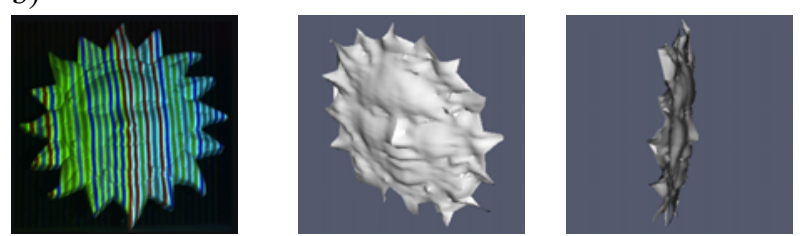

c)
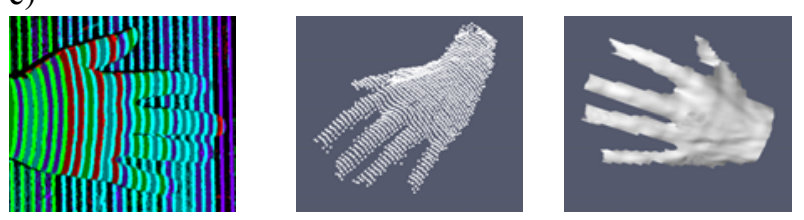

Fig. 5. Reconstruction tests.

\subsection{Conclusions of the new coding strategy}

A new way to encode one-shot stripe patterns has been presented. The new coding strategy is based on defining the pattern with a periodic binary luminance profile so that two adjacent stripes can share the same Hue value but not the same luminance value. The improvement with respect to encode a stripe pattern with a constrained De Bruijn sequence is that the resolution increases from $n(n-1)^{m-1}$ to $2 n^{m}$. In fact, $m$ is set to 3 so that in order to identify an stripe it is only necessary to read its own colour and the colours of the two adjacent stripes.

Some experimental results have been presented in order to show how easy is to segment the stripes and to demonstrate the validity of the technique.

\section{REFERENCES}

[1] K. L. Boyer and A. C. Kak. Color-encoded structured light for rapid active ranging. IEEE Transactions on Pattern Anal- 
ysis and Machine Intelligence, 9(1):14-28, 1987.

[2] B. Carrihill and R. Hummel. Experiments with the intensity ratio depth sensor. Computer Vision, Graphics and Image Processing, 32:337-358, 1985.

[3] D. Caspi, N. Kiryati, and J. Shamir. Range imaging with adaptive color structured light. Pattern Analysis and Machine Intelligence, 20(5):470-480, May 1998.

[4] C. Chen, Y. Hung, C. Chiang, and J. Wu. Range data acquisition using color structured lighting and stereo vision. Image and Vision Computing, 15:445-456, 1997.

[5] C. J. Davies and M. S. Nixon. A hough transform for detecting the location and orientation of 3-dimensional surfaces via color encoded spots. IEEE Transactions on systems, man and cybernetics, 28(1):90-95, February 1998.

[6] O. Faugeras. Three-Dimensional Computer Vision. MIT Press, 1993.

[7] P.M. Griffin, L.S. Narasimhan, and S.R. Yee. Generation of uniquely encoded light patterns for range data acquisition. Pattern Recognition, 25(6):609-616, 1992.

[8] Jens Gühring. Dense 3-d surface acquisition by structured light using off-the-shelf components. In Photonics West, Videometrics VII, volume 4309, pages 220-231, 2001.

[9] O. Hall-Holt and S. Rusinkiewicz. Stripe boundary codes for real-time structured-light range scanning of moving objects. In The 8th IEEE International Conference on Computer Vision, pages II: 359-366, 2001.

[10] E. Horn and N. Kiryati. Toward optimal structured light patterns. Image and Vision Computing, 17(2):87-97, February 1999.

[11] H. Hügli and G. Maître. Generation and use of color pseudo random sequences for coding structured light in active ranging. In Proceedings of Industrial Inspection, volume 1010, pages 75-82, Febrier 1989.

[12] M. Ito and A. Ishii. A three-level checkerboard pattern (tcp) projection method for curved surface measurement. Pattern Recognition, 28(1):27-40, 1995.

[13] R. Jarvis. Range sensing for computer vision. Advances in Image Communications. Elsevier Science Publishers, Amsterdam, pages 17-56, 1993.

[14] S. Kiyasu, H. Hoshino, K. Yano, and S. Fujimura. Measurement of the 3-D shape of specular polyhedrons using an m-array coded light source. IEEE Transactions on Instrumentation and Measurement, 44(3):775-778, June 1995.

[15] P. Lavoie, D. Ionescu, and E. Petriu. A high precision 3D object reconstruction method using a color coded grid and nurbs. In Proceedings of the International Conference on Image Analysis and Processing, pages 370-375, Venice, Italy, September 1999.

[16] F. J. MacWilliams and N. J. A. Sloane. Pseudorandom sequences and arrays. Proceedings of the IEEE, 64(12):17151729, 1976.

[17] M. Maruyama and S. Abe. Range sensing by projecting multiple slits with random cuts. Pattern Analysis and Machine Intelligence, 15(6):647-651, June 1993.
[18] M. Minou, T. Kanade, and T. Sakai. A method of time-coded parallel planes of light for depth measurement. Transactions of the IECE of Japan, 64:521-528, 81981.

[19] T. P. Monks, J. N. Carter, and C. H. Shadle. Colour-encoded structured light for digitisation of real-time 3D data. In Proceedings of the IEE 4th International Conference on Image Processing, pages 327-30, April 1992.

[20] R. A. Morano, C. Ozturk, R. Conn, S. Dubin, S. Zietz, and J. Nissanov. Structured light using pseudorandom codes. IEEE Pattern Analysis and Machine Intelligence, 20(3):322327, March 1998.

[21] H. Morita, K. Yajima, and S. Sakata. Reconstruction of surfaces of 3-d objects by m-array pattern projection method. In IEEE International Conference on Computer Vision, pages 468-473, 1988.

[22] E. M. Petriu, T. Bieseman, N. Trif, W. S. McMath, and S. K. Yeung. Visual object recognition using pseudo-random grid encoding. In Proceedings of the IEEE/RSJ International Conference on Intelligent Robots and Systems, pages 16171624, July 1992.

[23] J. L. Posdamer and M. D. Altschuler. Surface measurement by space-encoded projected beam systems. Computer Vision, Graphics and Image Processing, 18(1):1-17, 1982.

[24] J. Salvi, X Armangué, and J. Batlle. A comparative review of camera calibrating methods with accuracy evaluation. Pattern Recognition, 35(7):1617-1635, 2002.

[25] J. Salvi, J. Batlle, and E. Mouaddib. A robust-coded pattern projection for dynamic $3 \mathrm{~d}$ scene measurement. Pattern Recognition Letters, (19):1055-1065, September 1998.

[26] J. Salvi, J. Pagès, and J. Batlle. Pattern codification strategies in structured light systems. Pattern Recognition, 37(4):827849, 2004.

[27] H. J. W. Spoelder, F. M. Vos, E. M. Petriu, and Groen F. C. A. A study of the robustness of pseudo random binary array based surface characterization. In Proceedings of IEEE Instrumentation and Measurement Technology Conference, volume 2, pages 830-835, May 1997.

[28] J. Tajima and M. Iwakawa. 3-D data acquisition by rainbow range finder. In International Conference on Pattern Recognition, pages 309-313, 1990.

[29] R. J. Valkenburg and A. M. McIvor. Accurate 3d measurement using a structured light system. Image and Vision Computing, 16(2):99-110, February 1998.

[30] P. Vuylsteke and A. Oosterlinck. Range image acquisition with a single binary-encoded light pattern. Pattern Analysis and Machine Intelligence, 12(2):148-163, 1990.

[31] L. Zhang, B. Curless, and S. M. Seitz. Rapid shape acquisition using color structured light and multi-pass dynamic programming. In Int. Symposium on 3D Data Processing Visualization and Transmission, pages 24-36, Padova, Italy, June 2002. 